1 Using compact ${ }^{1} \mathrm{H}$ NMR@80 MHz and vibrational

$6 \quad{ }^{1}$ Niederrhein University of Applied Sciences, Frankenring 20, D-47798 Krefeld, Germany;

$9 \quad *$ corresponding author: martin.jaeger@hs-niederrhein.de 


\section{Supplemental Informations}

12 Selected constituents and additives and characteristic properties of commercially available

13 fuels according to ASTM D5599 and DIN EN ISO 22854 are RON, ethanol (EtOH), methyl-

14 tert-butyl ether (MTBE) and ethyl-tert-butyl ether (ETBE), oxygenates, olefins, aromatics,

15 benzene and oxygen. Representative values for the samples investigated in this study were

16 attributed to the corresponding commercial quality and listed in Table S1.

17 Table S1. RON and contents of selected major components and additives in fuel samples

18 classified according to the commercial fuel qualities. A total of four samples with a RON of

$1989,92,93$ and 104 are not listed in this table.

\begin{tabular}{cccccccccc}
\hline & \multicolumn{3}{c}{ Super } & \multicolumn{4}{c}{ Super Plus } & \multicolumn{3}{c}{ Premium } \\
\hline & min & mean & max & min & mean & max & min & mean & max \\
RON & 95.1 & 96.3 & 97.7 & 98 & 98.9 & 99.7 & 101.8 & 102.1 & 102.3 \\
MON & 85 & 85.1 & 85.3 & 88 & 88.1 & 88.2 & 88.9 & 89.5 & 90.3 \\
Ethanol $^{1}$ & 0.2 & 6.2 & 10 & 0 & 0.5 & 1 & 0.2 & 0.4 & 0.7 \\
ETBE $^{1}$ & 0 & 0.7 & 5.9 & 0 & 6.8 & 13.3 & 0.3 & 11.7 & 12.6 \\
MTBE $^{1}$ & 0 & 0.8 & 5.8 & 0 & 5.3 & 13.6 & 0 & 0.2 & 0.4 \\
Oxygenates $^{1}$ & 0 & 2.0 & 10.3 & 0 & 2.6 & 13.5 & 0 & 3.4 & 13.2 \\
Olefins $^{1}$ & 1.4 & 10.8 & 16.4 & 1.5 & 6.7 & 12.2 & 4.2 & 9.1 & 14.9 \\
Aromatics $^{1}$ & 18.8 & 29.6 & 34.9 & 21.3 & 28.5 & 35 & 31.9 & 34.0 & 34.9 \\
Benzene $^{1}$ & 0.2 & 0.7 & 0.9 & 0.2 & 0.5 & 0.8 & 0.1 & 0.2 & 0.3 \\
\hline
\end{tabular}

1) Vol.- $\%$. 

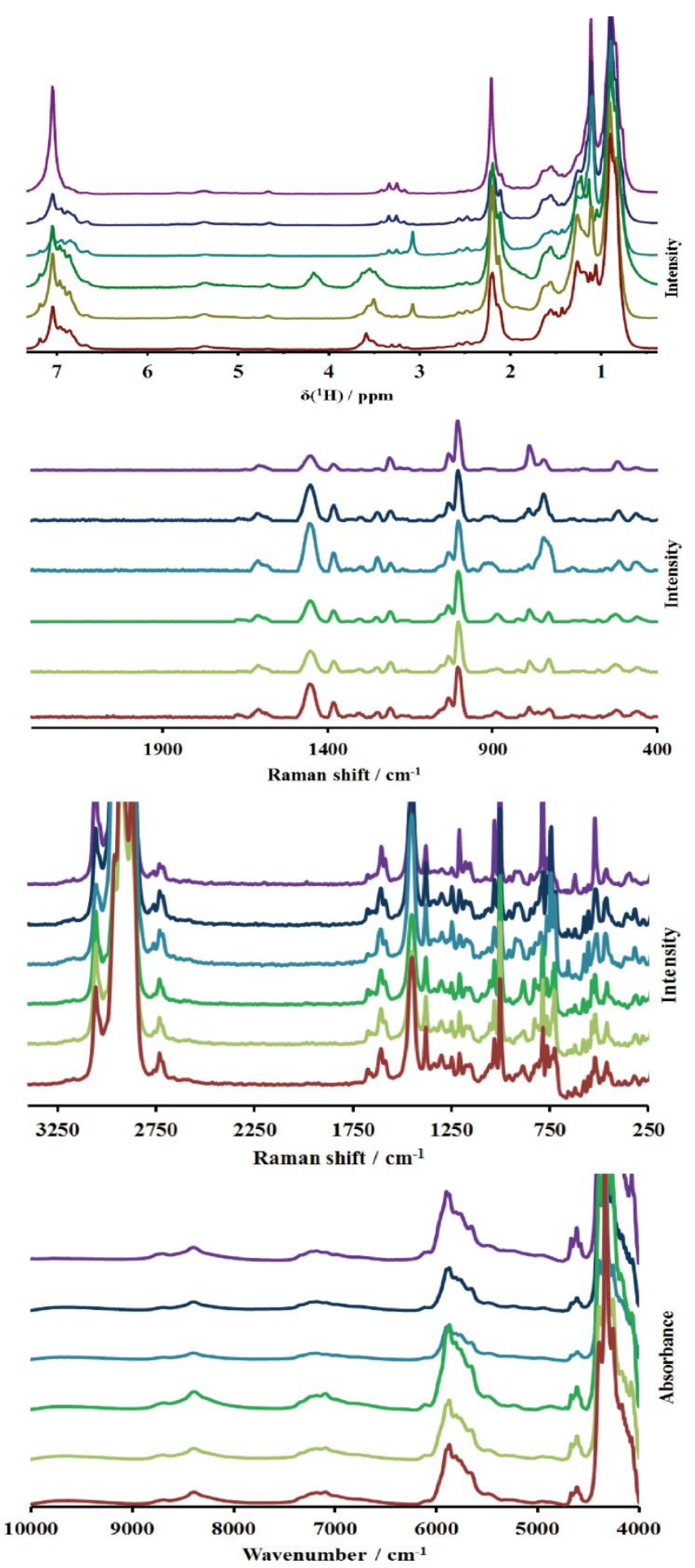

22 Fig. S1. From bottom to top: NIR, Ram, h-RAM and NMR spectra from gasoline samples,

23 with RON 95 (red), RON 96 (yellow), RON 97 (green), RON 98 (light blue), RON 99 (dark 24 blue), RON 102 (purple). 


\section{Precision of prediction}

27 Reproducibility and repeatability were used as measure to assess the goodness of RON 28 prediction. According to ASTM and DIN EN ISO norms, the terms refer to repetitive 29 measurements, thus replicates, while the absolute value of the CFR motor assay is considered 30 the true value. Spectra of replicates of fuel samples, i.e. triplicates, were recorded to 31 determine the RON using PLS and estimate repeatability and reproducibility, cf. Fig. S2. 32 While above-the-norm limits were used as a quality criterion for accuracy or trueness, here 33 the precision of the spectroscopic methods was evaluated.

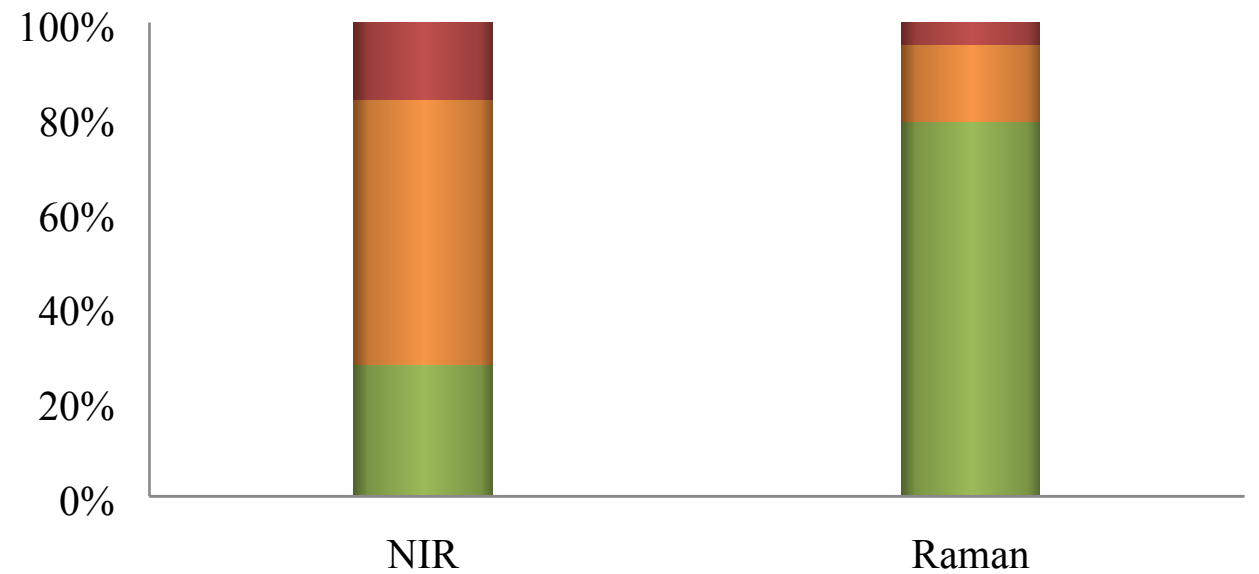

34 accuracy of the method. 
43 Concerning the precision, $79 \%$ of the samples reached the specified limits of reproducibility,

44 i.e. the standard deviation of the triplicate determination was lower than $0.2 .16 \%$ of the 45 samples fell within the repeatability limits, only $5 \%$ of the samples exceeded the acceptable 46 limits. Analogously to the accuracy, the precision of the NIR method was lower than that of 47 the Raman-based data, cf. Fig. S2. The outliers could be traced back to noisy spectra probably 48 from incident solar radiation when the samples were insufficiently shielded during 49 measurements. Comparison demonstrated that reproducibility and repeatability criteria within 50 a given technique were fulfilled to a higher degree than when using them as measure for the 51 goodness of RON prediction for the same technique, cf. Fig. 5. As expected, a replicate 52 spectroscopic prediction of RON is hence more precise than the accuracy of the prediction 53 itself. Nevertheless, reproducibility and repeatability provide ostensive means to estimate the 54 goodness of the prediction of RON via multivariate analysis of spectroscopic data. 

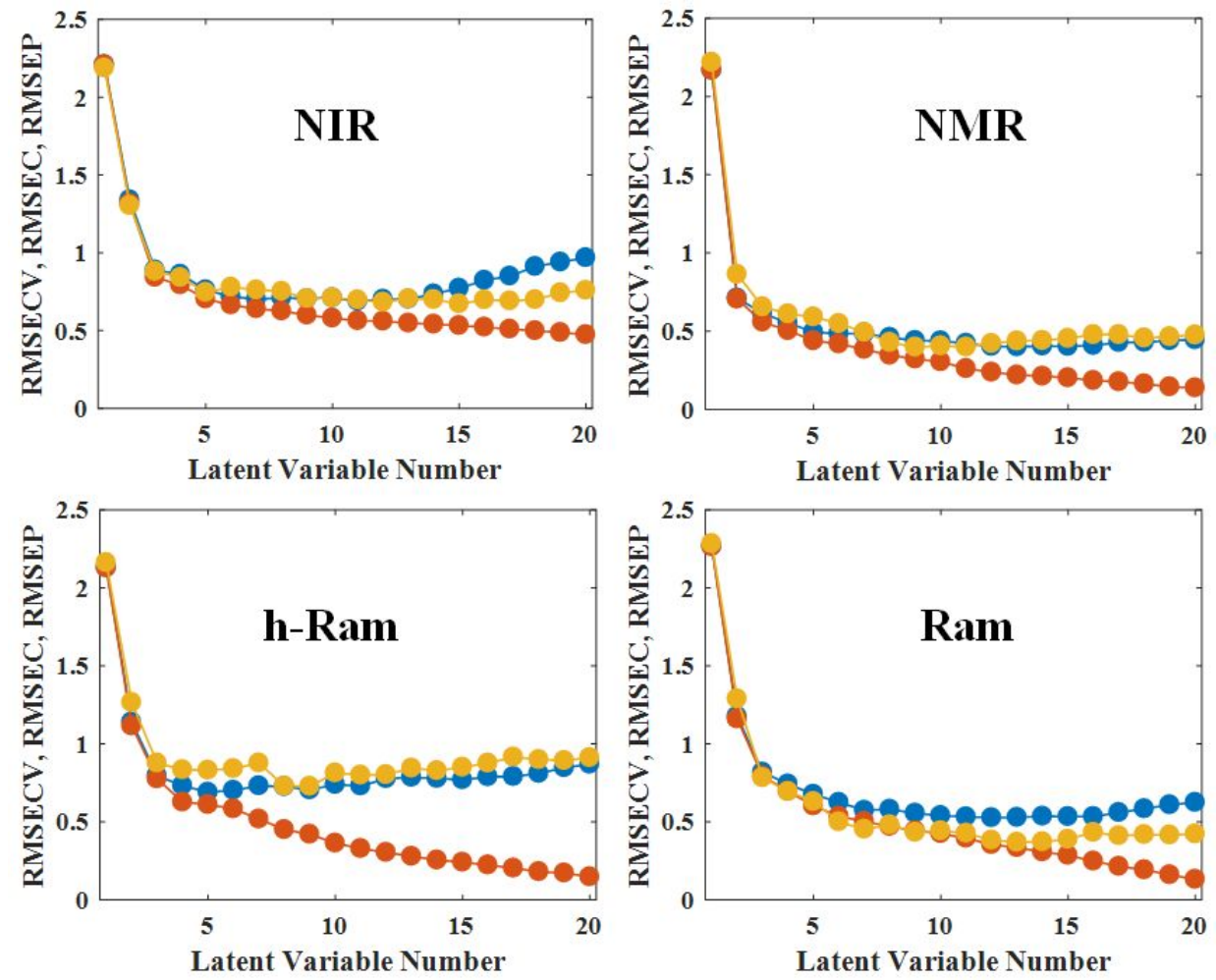

57 Fig. S3. RMSECV (blue), RMSEC (orange), RMSEP (yellow) depending on the number of 58 latent variables based on h-RAM (bottom, left), Ram (bottom, right), NIR (top, left) and 59 NMR80 (top, right) data and used in PLS regression for RON determination. 
62 Table S2 shows the influence of the choice of 4 and 9 latent variables on the multivariate

63 prediction of RON. Despite the choice of 9 latent variables, an overfitting did not occur, such

64 that the RMSEs indicated comparable results.

65 Table S2. RMSECV, RMSEP, bias and regression coefficient for the multivariate prediction 66 of RON based on the handheld Raman data with 4 and 9 latent variables.

\begin{tabular}{ccc}
\hline hRam & $\mathbf{4 ~ L V}$ & $\mathbf{9 ~ L V}$ \\
\hline RMSECV $^{1}$ & 0.73 & 0.7 \\
RMSEP $^{1}$ & 0.83 & 0.72 \\
Bias & -0.000076 & 0.00093 \\
$\mathbf{R}^{\mathbf{2}} \mathbf{P}$ & 0.83 & 0.87 \\
\hline
\end{tabular}

67 
Since the PLS-2 in combination with benchtop Raman data provided good results for RON and the four major additives, the prediction of further components in one model, such as olefin, aromatic, benzene and oxygenate contents, was attempted, see Fig. S4.

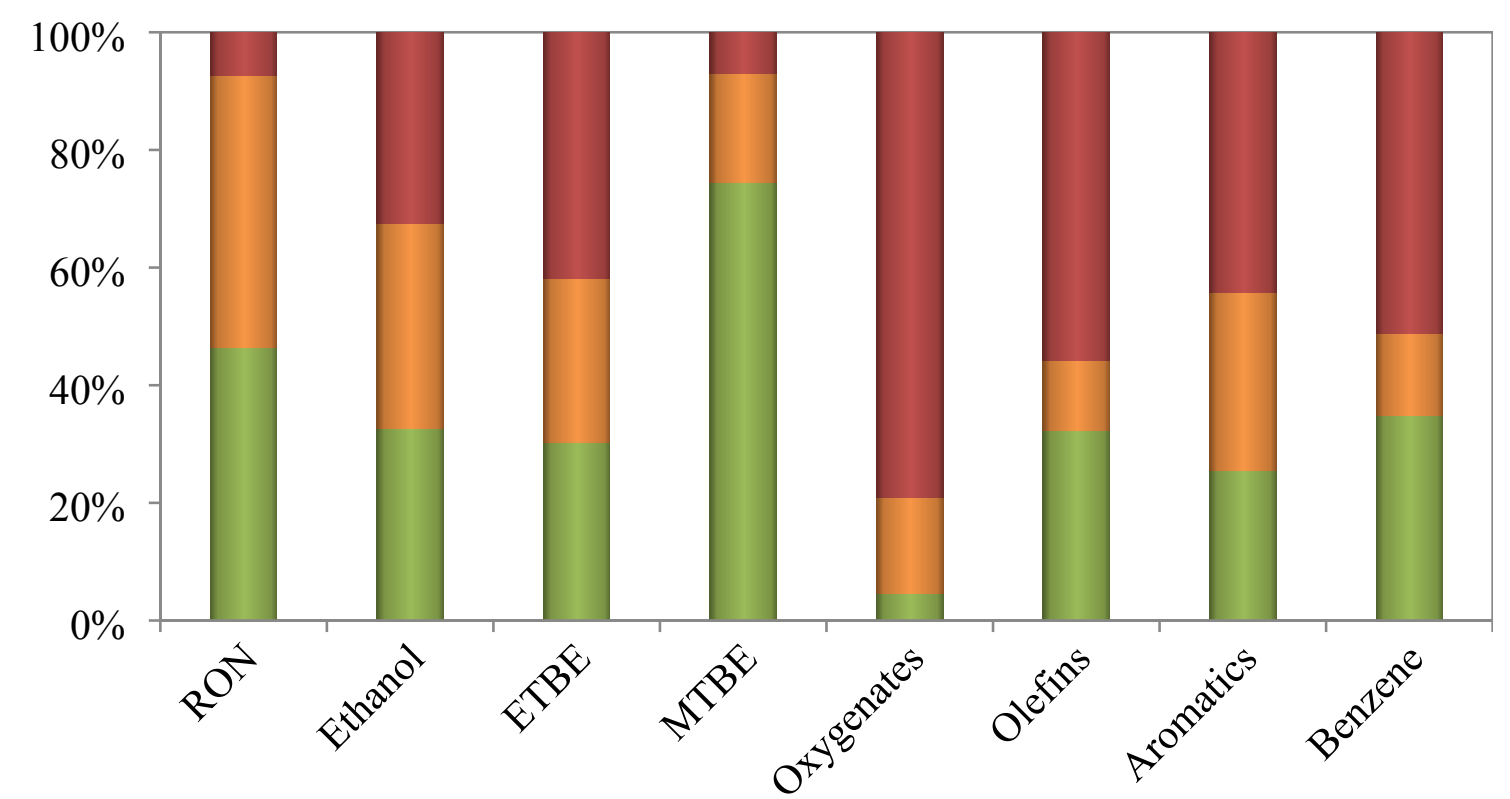

Fig. S4. Goodness of prediction of fuel parameters using PLS-2 based on benchtop Raman data and evaluated as reproducibility (green) and repeatability (orange) and outliers (red) according to norms.

The results prove that simultaneous prediction of all eight parameters using PLS-2 was feasible. Yet, the individual results varied significantly. While MTBE was predicted with high accuracy as was the RON, oxygenates and olefins were only poorly quantified. The differences in goodness between MTBE, ETBE, ethanol and oxygenates were found quite striking. This finding might be due to interdependencies or relationships of signals caused by the $\mathrm{C}-\mathrm{H}_{\mathrm{n}}-\mathrm{O}$ moieties. A high number of samples is another prerequisite for successful application of PLS-2. 
84 Hence, 120 samples for calibration model generation may just be too few, as the variations of 85 the crude oil in the refining process and the subsequent blending have an impact on the 86 spectra and hence on the composition itself. In contrast, PLS-2 has the time-saving and 87 simplicity advantage over successive calibration and model building for all desired parameters 88 individually. Yet, it does not necessarily apply to fuel samples and the characteristic values 89 due to strongly correlated parameters. Additive types and concentrations may vary for 90 samples with equal RON values, and depending on the number of samples used for the model 91 some may occur as outliers. Since density, oxygen and in some cases water content could not 92 be parameterized. This can be explained, e.g. in case of oxygen, as the absence of 93 corresponding signals. Density depends on temperature, which was not standardized for all 94 samples under investigation. In conclusion, a reasonable number of characteristic parameters 95 could be simultaneously estimated within acceptable limits using PLS-2. 